\title{
Efficacy and mechanism of combination of oxaliplatin with PKM2 knockdown in colorectal cancer
}

\author{
CHENXI YIN ${ }^{1,2^{*}}$, WENHUA LU ${ }^{1 *}$, MINGZHE MA $^{1}$, QIONG YANG $^{3}$, \\ WENZHUO $\mathrm{HE}^{1,4}$, YUMIN HU ${ }^{1}$ and LIANGPING XIA ${ }^{1,4}$ \\ ${ }^{1}$ State Key Laboratory of Oncology in South China, Sun Yat-sen University Cancer Center, \\ Collaborative Innovation Center for Cancer Medicine; ${ }^{2}$ Intensive Care Unit, \\ Sun Yat-sen University Cancer Center; ${ }^{3}$ Medical Oncology, Sun Yat-sen Memorial Hospital, Sun Yat-sen University; \\ ${ }^{4}$ VIP Region, Sun Yat-sen University Cancer Center, Guangzhou, Guangdong 510060, P.R. China
}

Received December 8, 2017; Accepted October 23, 2019

DOI: $10.3892 / \mathrm{ol} .2020 .12175$

\begin{abstract}
M2 isomer of pyruvate kinase (PKM2), a key enzyme in aerobic glycolysis, is closely related to cancer development and progression. Suppression of PKM2 exhibits synergistic effects with docetaxel in lung cancer, but the therapeutic potential in colorectal cancer (CRC) is unclear. The aim of the present study was to explore the synergic effects and mechanism of knocking down PKM2 combined with oxaliplatin (a chemosensitizer) treatment in two CRC cell lines (HCT116 and DLD1). The PKM2 gene was initially knocked down using small interfering (si)RNAs (si155 and si156). Subsequently, the effects of PKM2-siRNAs and oxaliplatin, on CRC cells were determined using MTS, cell cycle analysis and apoptosis assays. The mechanism of targeting PKM2 was explored by detecting glucose uptake, lactate secretion fluxes, and the levels of glucose-6-phosphate dehydrogenase (G6PD) mRNA, glutathione (GSH) and reactive oxygen species (ROS). Cell viability in the experimental groups (PKM2-siRNAs, oxaliplatin, PKM2-siRNAs + oxaliplatin) was significantly reduced compared with the control group, and combination treatments (PKM2-siRNAs + oxaliplatin) were more effective than single treatments (PKM2-siRNAs and oxaliplatin only groups). Similar results were observed with the apoptosis assay. The combination groups showed synergistic effects compared with both single treatment groups. Furthermore,
\end{abstract}

Correspondence to: Dr Yumin Hu or Dr Liangping Xia, State Key Laboratory of Oncology in South China, Sun Yat-sen University Cancer Center, Collaborative Innovation Center for Cancer Medicine, 651 Dongfeng East Road, Guangzhou, Guangdong 510060, P.R. China

E-mail: huym@sysucc.org.cn

E-mail: xialiangping@163.com

${ }^{*}$ Contributed equally

Key words: M2 isomer of pyruvate kinase, oxaliplatin, aerobic glycolysis, reactive oxygen species, pentose phosphate pathway, colorectal cancer glucose uptake and lactate secretion and mRNA levels of G6PD and PKM2 were decreased after PKM2 knockdown in the PKM2-siRNAs and PKM2-siRNAs + oxaliplatin groups. The GSH levels in the PKM2-siRNAs group was significantly lower compared with the negative control group. The ROS levels in the PKM2-siRNAs groups were also significantly increased. The combination of PKM2-siRNAs and oxaliplatin had synergistic effects on CRC cells (HCT116 and DLD1). PKM2 silencing may alter energy metabolism in cancer cells and initiate ROS-induced apoptosis after downregulation of the pentose phosphate pathway by PKM2-siRNAs.

\section{Introduction}

Energy metabolism of cancer cells predominantly involves aerobic glycolysis, in contrast to normal differentiated cells, which rely primarily on mitochondrial oxidative phosphorylation to generate the energy needed for cellular processes $(1,2)$. To adapt to the hypoxic microenvironment and compete with surrounding normal cells for limited resources, cancer cells utilize aerobic glycolysis, converting most glucose to lactate regardless of whether oxygen is present, to ensure rapid proliferation $(1,3)$. Although the efficiency of aerobic glycolysis for energy production is very low, the rate is extremely high (1). Pyruvate kinase (PK) is a key enzyme involved in aerobic glycolysis and exists as four isomers: L, R, M1 and M2. PKL and PKR are expressed in hepatic cells and red blood cells, respectively. PKM1 is expressed in most mature cells (4). The M2 isomer of pyruvate kinase (PKM2) is expressed in cancer cells and tissues, which has a strong ability to promote cell proliferation and serves a crucial role in tumor development and survival (4). PKM2 is a driver enzyme of aerobic glycolysis and a hotspot of present studies in the field of tumor metabolism (5-7). PKM2 primarily exists in an inactive dimer form in the tumor, catalyzing the conversion of pyruvic acid. This accumulates upstream glycolytic intermediates as an anabolic supply for the synthesis of lipids and nucleic acids, which promotes the anabolism of bio-macromolecules. The tetramer of PKM2 is activated and catalyzes the conversion of pyruvic acid to ATP. The subtype of PKM2 is determined by its phosphorylation status, which mediates glucose conversion (8). 
Kress et al (9) found that mRNA levels of PKM2 in colorectal cancer (CRC) were significantly higher compared with normal tissues. Subsequently, a meta-analysis by Kumar et al (10) showed similar results. They also found that serum PKM2 protein levels in patients with gastrointestinal tumors and esophageal cancer were higher compared with healthy patients. PKM2 may be used for tumor diagnosis and shows similar sensitivity to the classic marker carcinoembryonic antigen in CRC $(11,12)$, and meta-analyses by Zhang et al and Hathurusinghe et al $(12,13)$ also demonstrated that PKM2 was gradually increased with the degree of malignancy of the disease. Furthermore, the diagnostic and prognostic value of PKM2 has also been demonstrated in other types of tumors, including pancreatic cancer (14), cervical cancer (15), lung cancer (16), renal cancer (17), melanoma (18) and breast cancer $(19,20)$.

Shi et al (21) found that PKM2 suppression reduced tumor growth and also exhibited synergistic effects with docetaxel in A549 lung cancer cells. In addition, Lin et al (20) revealed high PKM2 expression was significantly associated with in vitro chemosensitivity to epirubicin and 5-fluorouracil (5-Fu) in patients with breast cancer. However, studies on the association between PKM2 and chemotherapy are limited in metastatic CRC $(22,23)$. A previous study revealed that the response rate to oxaliplatin was reduced in the PKM2 downregulated HTOXAR3 cell line of CRC compared with its parental cell line HT29, which was also validated in clinical settings (22).

Currently, oxaliplatin-based regimens are the most effective treatment for CRC $(24,25)$. Efforts aimed at improving the efficacy of this regimen may result in improved outcomes (22). The mechanism of oxaliplatin action is mediated by the formation of DNA adducts, which induce DNA lesions such as intrastrand crosslinks by covalently binding the platinum compound to guanine residues. Oxaliplatin DNA adducts are thought to exert their cytotoxicity by directly inhibiting DNA and RNA synthesis and inducing apoptosis (26). The cytotoxic effects of oxaliplatin in CRC cell lines involve the p53 gene status via induced activation of the p53-p21 pathway (23). Whether the combination treatment of PKM 2 knockdown and oxaliplatin has a synergistic effect in CRC is yet to be elucidated. The aim of the present study was to determine whether PKM2 and oxaliplatin exhibited synergistic effects and to evaluate the potential mechanism by which PKM2 induced apoptosis. The results may be valuable in developing novel treatment approaches targeting PKM2.

\section{Materials and methods}

Cell lines and cell culture. The human CRC cell lines HCT116 and DLD1 were purchased from the Cell Bank of Chinese Academy of Sciences and cultured in McCoy's 5A and RPMI-1640 medium, respectively, both supplemented with 10\% fetal bovine serum (Gibco; Thermo Fisher Scientific, Inc.) in humidified conditions with $5 \% \mathrm{CO}_{2}$ at $37^{\circ} \mathrm{C}$.

Small interfering (si)RNA transfection. CRC cells (HCT116 and DLD1) were transfected with siRNA duplex oligonucleotides targeting PKM2 (50 nM) using Lipofectamine ${ }^{\mathrm{TM}}$ RNAiMAX transfection reagent (cat. no. 13778150,
Thermo Fisher Scientific, Inc.) according to the manufacturer's protocol. Cells were incubated for $24 \mathrm{~h}$ at $37^{\circ} \mathrm{C}$ after transfection. Western blot analysis was performed to determine the knockdown efficiency of PKM2-siRNAs. The sequences of the PKM2 siRNAs were: si155, 5'-GCCAUAAUCGUCCUCACCA-3'; si156, 5'-CCAUAAUCGUCCUCACCAA-3'; and si27, 5'-AGC AGAGCUGCAUCUA-3' according to a previous study (27). Additionally, an siRNA with no influence on PKM2 function was used as a negative control (NC, 5'-CUUACGCUGAGU ACUUCGA-3').

Western blot analysis of PKM2 and lactate dehydrogenase $(\mathrm{LDH})$ expression. Transfected cells were washed twice with cold PBS and harvested in lysis buffer (5\% sodium dodecyl sulfate, $10 \mathrm{mM}$ EDTA, $50 \mathrm{mM} \mathrm{NaCl}, 10 \mathrm{mM}$ Tris- $\mathrm{HCl}$ ). A Pierce bicinchoninic acid protein assay kit (Thermo Fisher Scientific, Inc.) was used to determine protein concentrations. Subsequently, $50 \mu \mathrm{g}$ each sample was electrophoresed using $10 \%$ SDS-PAGE and transferred to $0.45 \mu \mathrm{m}$ PVDF, sealed using 5\% milk at room temperature for $1 \mathrm{~h}$ and incubated with antibodies (EMD Millipore). Membranes were probed with anti-PKM2, anti-LDH and anti-GAPDH rabbit antibodies (cat. nos. 4053, 3582 and 5174, respectively; all 1:1,500; Cell Signaling Technology, Inc.). The primary antibody was incubated at $4^{\circ} \mathrm{C}$ overnight, and the secondary antibody was incubated at room temperature for $2 \mathrm{~h}$. Protein expression was normalized against $\beta$-actin expression (cat. no. 4970; 1:1,000; Cell Signaling Technology, Inc.) and signal were visualized using an enhanced chemiluminescence kit (Thermo Fischer Scientific, Inc.).

Cell viability analysis. Cells were seeded at the density of $3 \times 10^{3}$ cells/well in $100 \mu \mathrm{l}$ culture medium in a 96-well plate. Following overnight incubation, culture medium was added to the experimental (si155, si156, oxaliplatin (3 $\mu \mathrm{mol} / \mathrm{l})$, si155 + oxaliplatin and si156 + oxaliplatin) or control cells (NC), respectively. Si155 and si156 groups were added to the same amount of medium as oxaliplatin. To determine the activity of the cells, cells were imaged $24 \mathrm{~h}$ after oxaliplatin treatment during the logarithmic growth phase. An MTS assay was performed at 24, 48, 72 and $96 \mathrm{~h}$. For this assay, $20 \mu \mathrm{l} \mathrm{MTS}$ solution (Promega Corporation) was added to each well and the cells were incubated at $37^{\circ} \mathrm{C}$ for $4 \mathrm{~h}$ before the absorbance was determined using a MultiSkan microplate reader (Thermo Fisher Scientific, Inc.) at a wavelength of $490 \mathrm{~nm}$.

Apoptosis analysis. HCT116 and DLD1 CRC cells were seeded into a 6 -well plate at a density of $1 \times 10^{5}$ cells/well. A total of $48 \mathrm{~h}$ after transfection, both attached and floating cells were harvested and washed twice with PBS. Cells were resuspended and stained using an annexin-V/PI assay kit (Nanjing KeyGen Biotech Co., Ltd.) as previously described (23). Cell mortality was determined using a flow cytometer (Beckman Coulter, Inc.).

Glucose uptake and lactate secretion assays. Per protein extracellular fluxes, including glucose/glutamine uptake and lactate/glutamate secretion of HCT116 and DLD1 CRC cells were calculated by subtracting the substrate concentrations in the final spent medium from those in the initial medium 
using a Yellow Springs Instrument (YSI) 2950 biochemistry analyzer and the YSI 2776 glucose/lactate standard $(2.5 \mathrm{~g} / 1$ glucose, $0.5 \mathrm{~g} / 1$ lactate; YSI; Xylem, Inc.) (28). Cells in the logarithmic phase were seeded into 6-well plates at a density of $2 \times 10^{5}$ cells/well. Following overnight incubation, culture medium (NC, si155, si156, oxaliplatin, si155 + oxaliplatin and si156 + oxaliplatin) were added and the plates were incubated for $24 \mathrm{~h}$ and culture medium collected for detection and normalized to the cell numbers after $24 \mathrm{~h}$.

Reverse-transcription-quantitative (RT-q)PCR. Total RNA was extracted from HCT116 CRC cells using TRIzol ${ }^{\circledR}$ (Invitrogen; Thermo Fisher Scientific, Inc.) according to the manufacturer's instructions. cDNA was synthesized using the MLV transcriptase kit (Invitrogen; Thermo Fisher Scientific, Inc.). Fast SYBR Green master mix was used to determine the threshold cycle (Cq) value of each sample using a CFX96 real-time quantitative PCR detection system (Bio-Rad Laboratories, Inc.). GAPDH served as the gene used for normalization. The fold-changes were calculated using the relative quantification with $2^{-\Delta \Delta C q}(29)$. All reactions were performed in a $20 \mu \mathrm{l}$ reaction volume in triplicate. The following PCR conditions were used: Initial denaturation at $95^{\circ} \mathrm{C}$ for $30 \mathrm{sec}$; followed by 40 cycles of $95^{\circ} \mathrm{C}$ for $5 \mathrm{sec}$ and $60^{\circ} \mathrm{C}$ for $30-60 \mathrm{sec}$; and stage 3 was dissociation according to the manufacturer's protocol (cat. no. RR420; Takara Biotechnology Co., Ltd.).

The PCR primer sequences used were as follows: GAPDH forward, 5'-AAGGTCATCCCTGAGCTGAA-3' and reverse, 5'-TGACAAAGTGGTCGTTGAGG-3'; G6PD forward, 5'-TGCATGAGCCAGATAGGCTG-3' and reverse, 5'-GGT AGTGGTCGATGCGGTAG-3'; and PKM2 forward, 5'-ATG CAGCACCTGATAGCTCG-3' and reverse, 5'-AGGCTCGCA CAAGTTCTTCA-3'.

Analysis of cellular glutathione (GSH) levels. GSH was measured using the GSH-Glo ${ }^{\mathrm{TM}}$ glutathione assay kit (cat. no. V6911; Promega Corporation) according to the manufacturer's instructions. Briefly, HCT116 cells were seeded at a density of $2 \times 10^{4}$ cells/well into 96 -well opaque plates and treated with the indicated siRNA (si155 or si156). After removing the medium, the cells were incubated in $100 \mu \mathrm{l}$ mixed GSH-Glo ${ }^{\mathrm{TM}}$ reagent for $35 \mathrm{~min}$ at room temperature and subsequently in $100 \mu 1$ reconstituted Luciferin Detection Reagent for $15 \mathrm{~min}$ at room temperature. Luminescent signals were detected using a Fluoroskan luminescence scanner (Thermo Fisher Scientific, Inc.).

Analysis of reactive oxygen species (ROS). After treatment with PKM2-siRNAs (si155 and si156) or scrambled siRNAs for $24 \mathrm{~h}$, HCT116 cells were incubated with $10 \mu \mathrm{M}$ 2',7'-dichlorofluorescin diacetate (Sigma-Aldrich; Merck $\mathrm{KGaA}$ ) for $30 \mathrm{~min}$, followed by flow cytometry analysis using a FACSCalibur flow cytometer (BD Biosciences).

Statistical analysis. Data were presented as mean \pm SD. Differences between two groups were compared using unpaired Student's t-test and differences between multiple groups were compared using LSD or Tukey's post-hoc test following one-way ANOVA test for comparisons of 3 groups or more respectively. $\mathrm{P}<0.05$ was considered to indicate a statistically significant difference. GraphPad Prism 7 software (GraphPad Software, Inc.) was used for calculating these statistics.

\section{Results}

Determining the validity of candidate PKM2-siRNAs. To explore the chemosensitization effects of targeting PKM2 in CRC cells, the PKM2 gene was initially knocked down using siRNAs. The si-PKM2 sequences were specifically synthesized as described by Goldberg et al (27). The most efficient sequences, si27, si155, and si156, targeting PKM2 were selected, and si-NC was used the negative control. The knockdown efficiency of PKM2-siRNAs (si27, si155, si156) was verified using western blot analysis. Protein level of PKM2 was analyzed in two colorectal cancer (CRC) cell lines (HCT116 and DLD1). PKM2 protein expression was reduced after transfection of si155 and si156 in CRC cells (HCT116 and DLD1) compared with si-NC (Fig. 1A). The results indicated that si155 and si156 were the most effective siRNAs for knockdown of PKM2 in CRC cells, and thus these siRNAs were used to downregulate PKM2 in subsequent analyses.

Effects of PKM2 knockdown on proliferation and apoptosis of CRC cells. si155 and si156 were transfected into HCT116 and DLD1 cell lines and the effects on cell-proliferation was evaluated using an MTS assay at different time points $(24,48,72$ and $96 \mathrm{~h})$. The results showed that both siRNAs significantly attenuated the proliferative ability of CRC cells (Fig. 1B). Consistently, similar results were also observed with the apoptosis assay. Transfection of siRNAs resulted in increased apoptosis in CRC cells compared with the NC group (Fig. 1C and D).

In order to examine the effect of PKM2-siRNAs on cell viability of HCT116 and DLD1, the logarithmic growth of HCT116 and DLD1 cells was observed in different experimental groups (NC, PKM2-siRNAs and PKM2-siRNAs + oxaliplatin; Fig. 2).

Synergistic anti-proliferative and apoptotic effects of PKM2-siRNAs and oxaliplatin in vitro. As oxaliplatin-based chemotherapy is not only a traditional but also an effective regimen in CRC (30), to investigate whether PKM2-siRNAs augmented the antitumor effects of oxaliplatin, proliferation and apoptosis assays were performed.

Cell proliferation was evaluated using an MTS assay in the PKM2-siRNAs + oxaliplatin, PKM2-siRNAs only, oxaliplatin only and NC groups. Cellular proliferation was significantly inhibited in the PKM2-siRNAs + oxaliplatin group compared with the PKM2-siRNAs or the oxaliplatin only group. Additionally, treatment with oxaliplatin or PKM2-siRNAs alone showed cytotoxic effects compared to in the NC group (Fig. 3A and B).

The number of apoptotic cells was analyzed using flow cytometry. As shown in Fig. 3C and D, treatment with PKM2-siRNAs + oxaliplatin resulted in a synergistic increase in apoptosis compared with oxaliplatin or PKM2-siRNAs alone.

Keyfactors relating to apoptosis induced by PKM2 knockdown. Cancer cells obtain energy and also maintain a stable state 
A

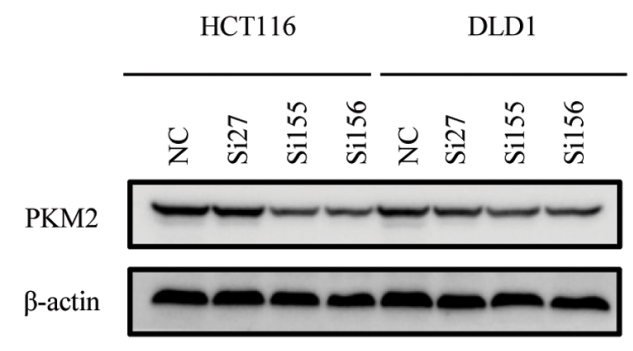

B

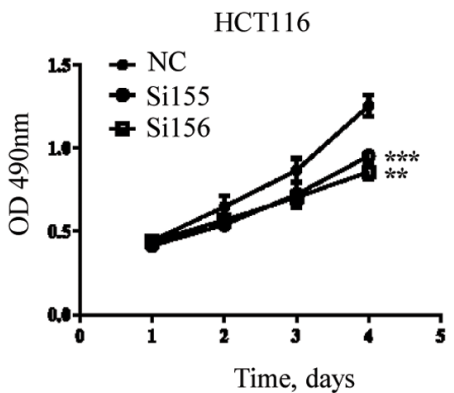

DLD1

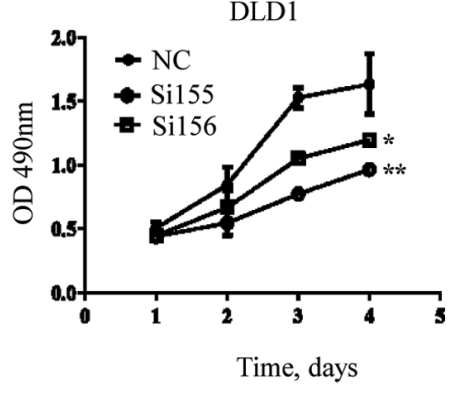

C

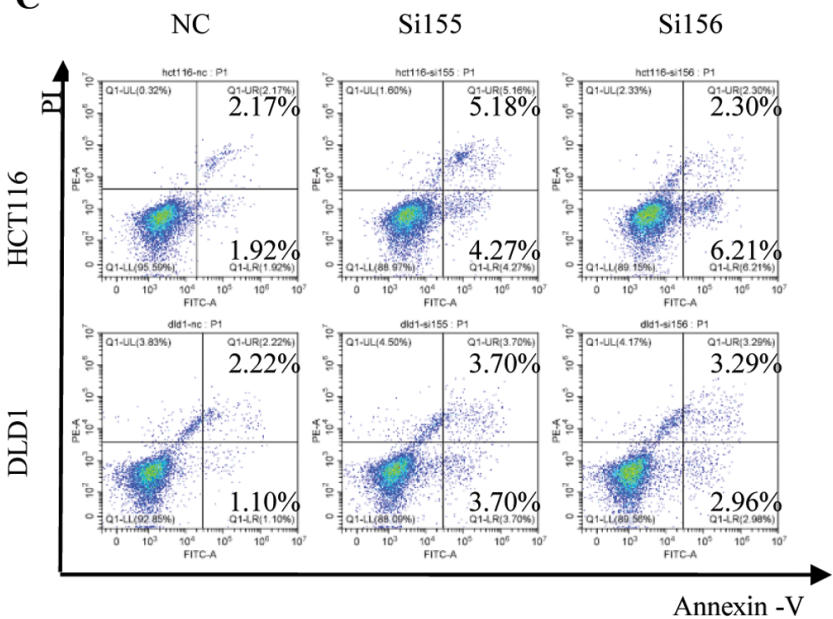

D

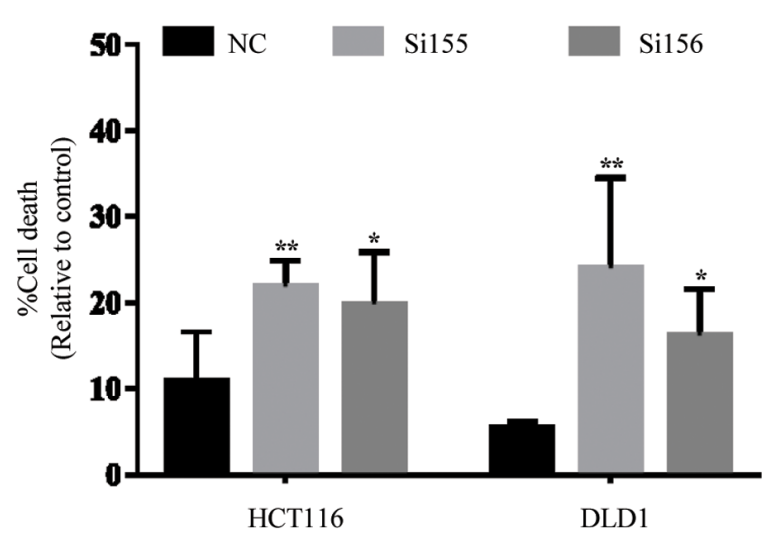

Figure 1. Efficiency of knockdown with different PKM2-siRNAs and their effects on proliferation and apoptosis of HCT116 and DLD1 cells. (A) Western blot analysis of NC and PKM2-siRNAs (si155, si156, and si27). (B) The effect of PKM2-siRNAs (si155 and si156) on proliferation of HCT116 and DLD1 cells. Viability of the si155 and si156 groups were significantly reduced. (C) Flow cytometry plots and (D) quantitative analysis of apoptosis in HCT116 and DLD1 cells transfected with PKM2-siRNAs. Apoptosis of PKM2-siRNAs groups was increased compared with that in the NC group. ${ }^{*} \mathrm{P}<0.05,{ }^{* *} \mathrm{P}<0.01,{ }^{* * * *} \mathrm{P}<0.001$ vs. respective NC. NC, negative control; PKM2, M2 isomer of pyruvate kinase; siRNA, small interfering RNA.

A

NC
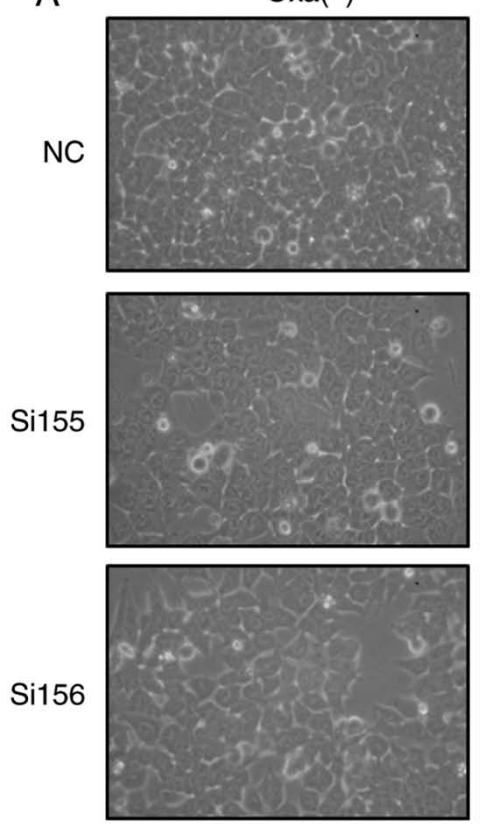

HCT116 (×20)
B
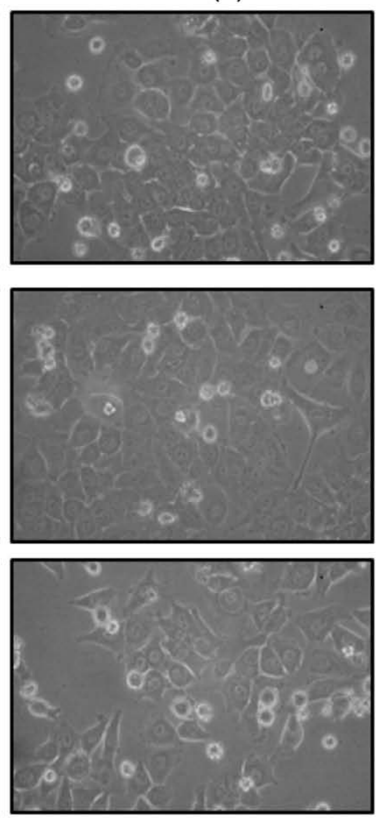
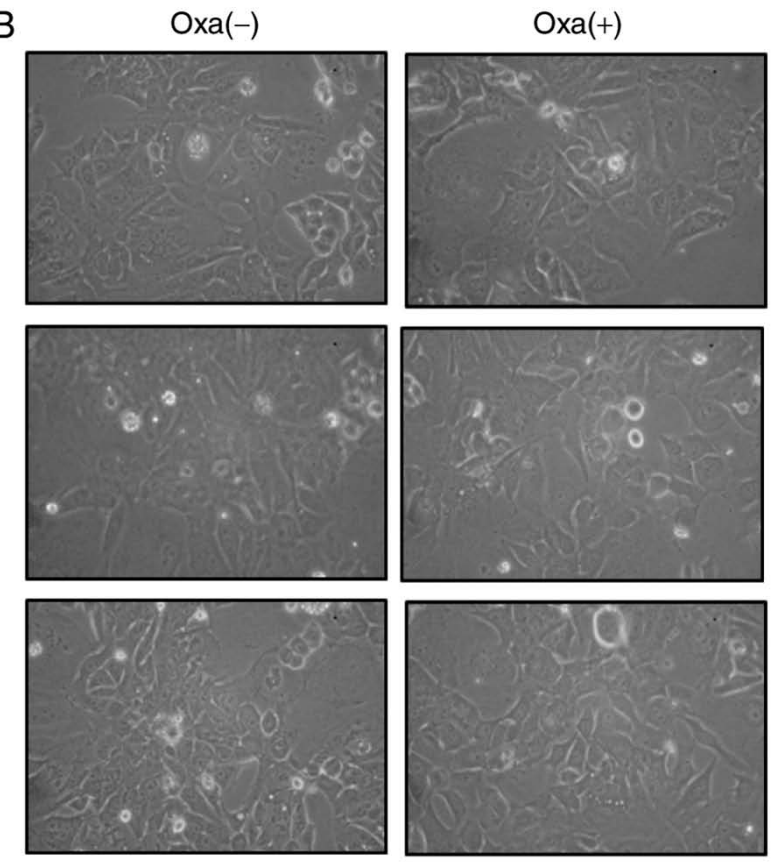

DLD1 $(\times 20)$

Figure 2. Images of (A) HCT116 and (B) DLD1 cells (NC, PKM2-siRNAs and PKM2-siRNAs + oxa) in the logarithmic growth phase. Images were taken $24 \mathrm{~h}$ after the cells were treated with or without oxa. NC, negative control; oxa; oxaliplatin; siRNA, small interring RNA; -, without oxa; +, with oxa; PKM2, M2 isomer of pyruvate kinase. 
A
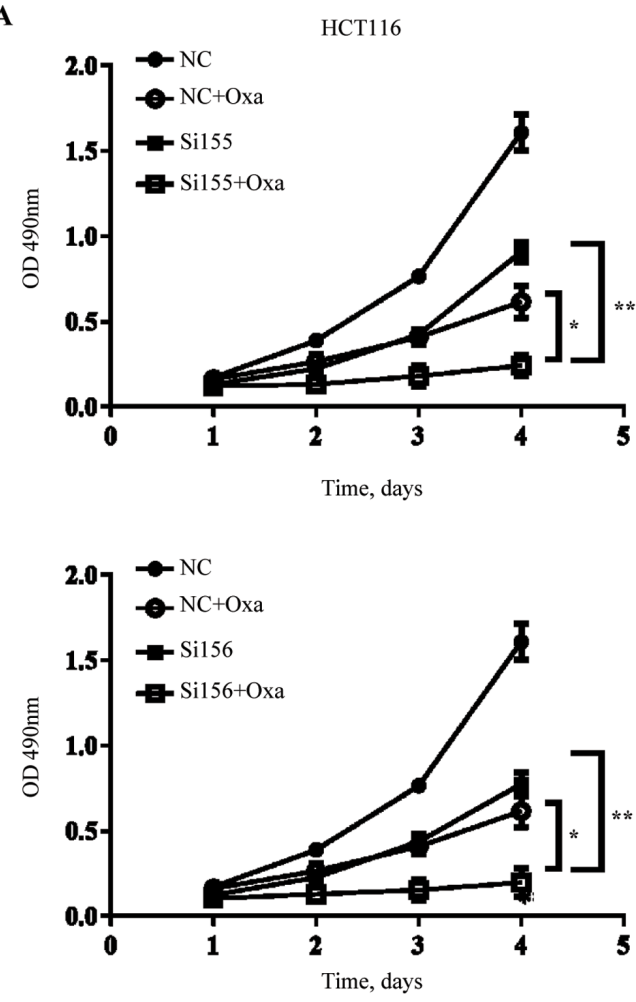

C

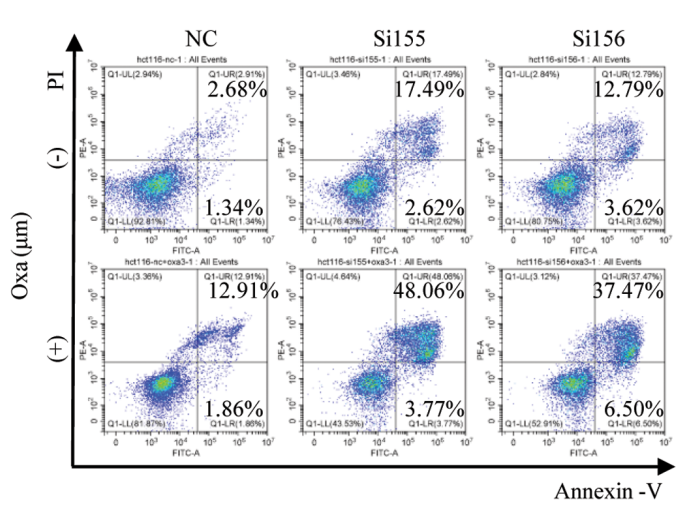

D

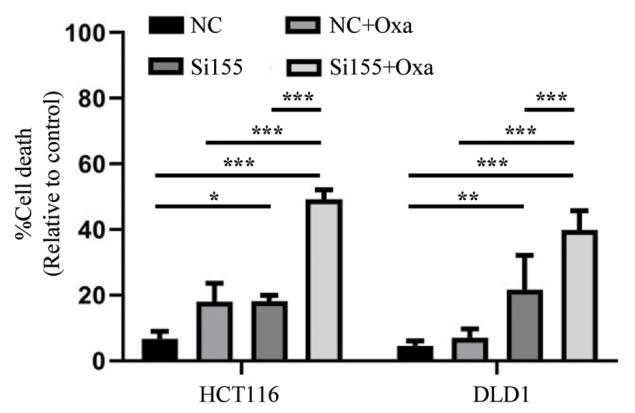

B
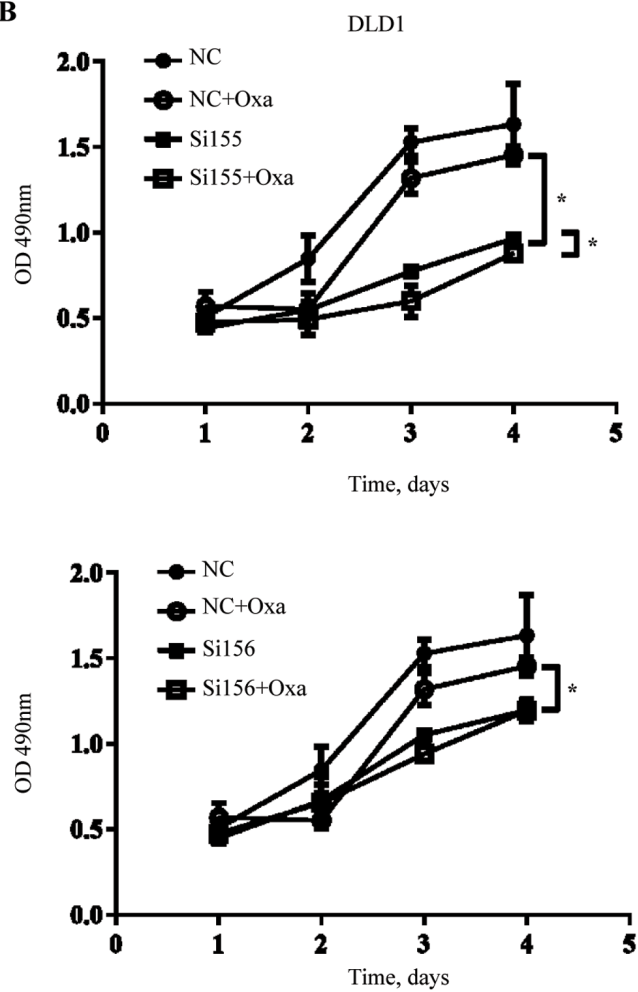

DLD1
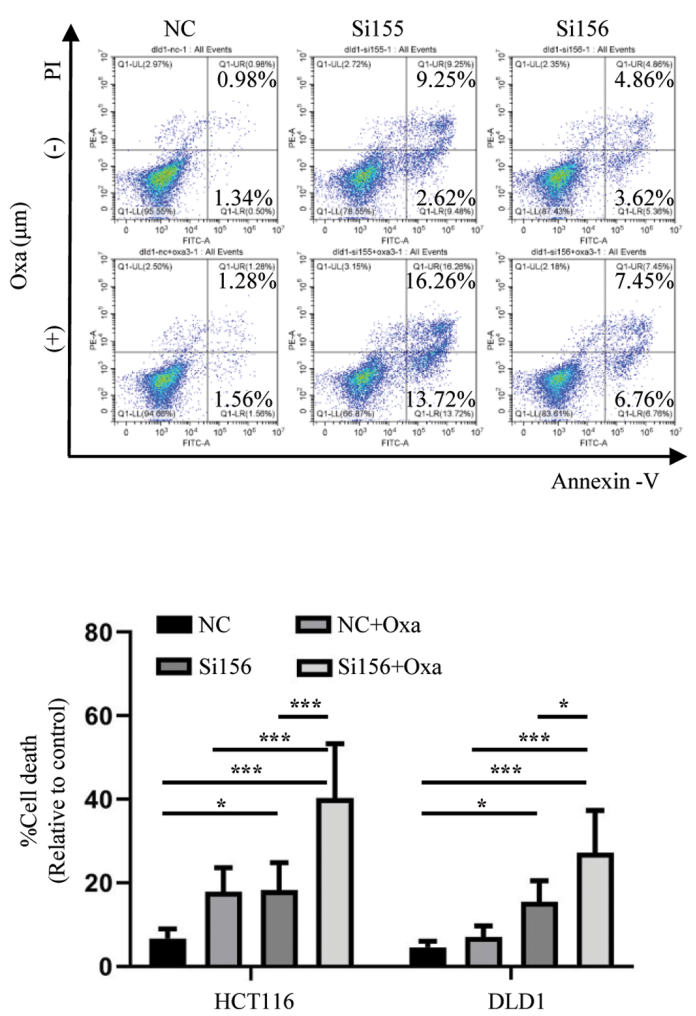

Figure 3. Synergistic anti-proliferative and apoptotic effects of PKM2-siRNAs transfection and Oxa treatment in HCT116 and DLD1cells. Cell proliferation of (A) HCT116 and (B) DLD1 cells with and without PKM2-siRNAs and Oxa treatment. (C) Flow cytometry plots and (D) respective quantitative analysis of apoptosis of HCT116 and DLD1 cells with and without PKM2-siRNAs transfection and oxa treatment. ${ }^{*} \mathrm{P}<0.05,{ }^{* * *} \mathrm{P}<0.01,{ }^{* * * *} \mathrm{P}<0.001$ for indicated comparison. PKM2, M2 isomer of pyruvate kinase; siRNA, small interfering RNA; oxa, oxaliplatin; NC, negative control.

through glycolysis (1,3). Glucose uptake and lactate secretion fluxes of the NC, PKM2-siRNAs and PKM2-siRNAs + oxaliplatin treated HCT116 and DLD1 cells were measured.
PKM2 knockdown reduced the protein expression of lactate dehydrogenase (LDH), glucose uptake and lactate secretion in the PKM2-siRNAs and PKM2-siRNAs + oxaliplatin groups 
$\mathbf{A}$

HCT116

$$
\text { NC } \quad \text { Si155 Si156 NC Si155 Si156 }
$$

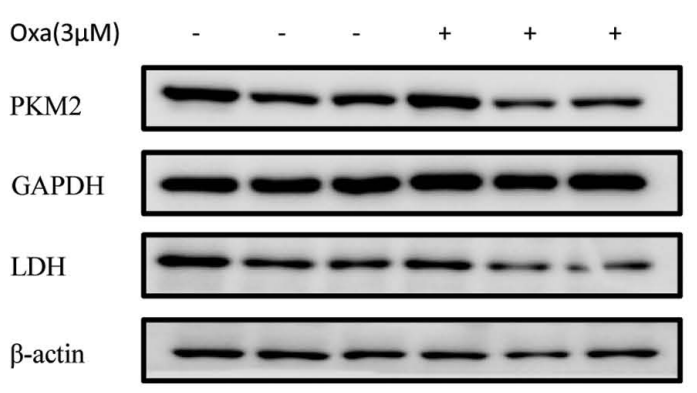

$\mathbf{B}$
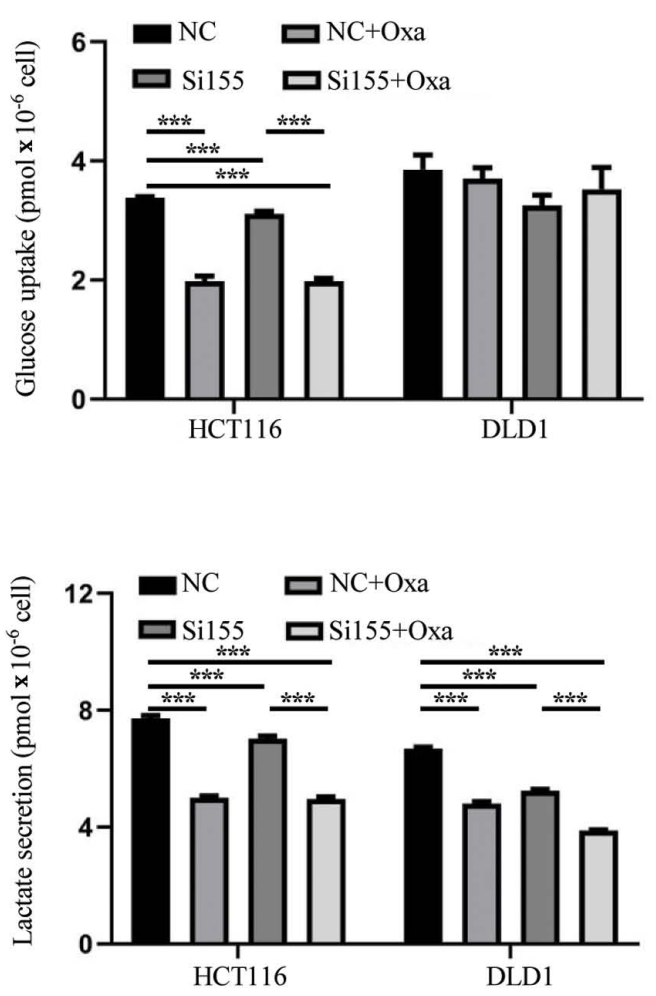

DLD1

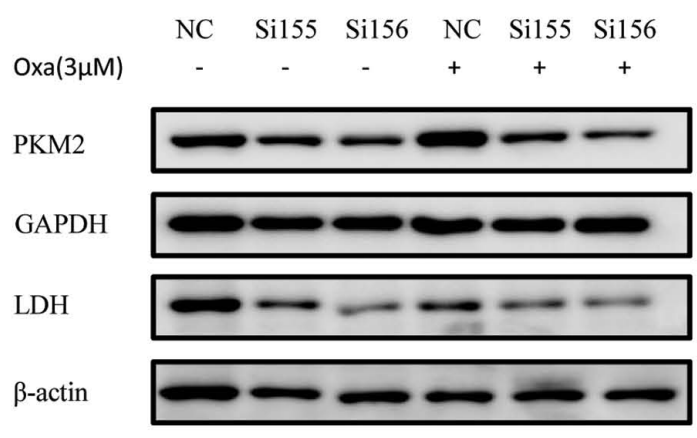

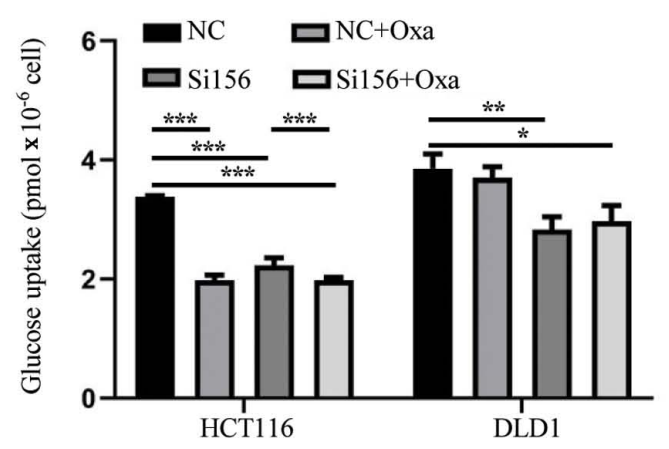

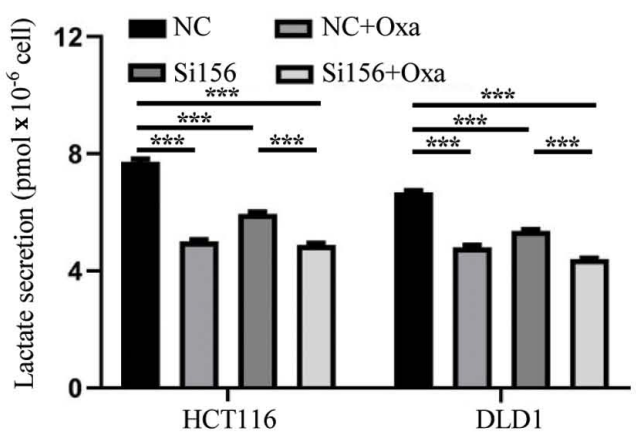

Figure 4. Glucose uptake and lactate secretion fluxes of the NC, PKM2-siRNAs and PKM2-siRNAs + oxaliplatin treated HCT116 and DLD1 cells. (A) Western blot analysis of PKM2 and LDH and (B) glucose uptake and lactate secretion fluxes of NC, PKM2-siRNAs and PKM2-siRNAs + oxaliplatin experimental groups in HCT116 and DLD1 cells. ${ }^{*} \mathrm{P}<0.05,{ }^{* *} \mathrm{P}<0.01,{ }^{* * *} \mathrm{P}<0.001$ for indicated comparison. $\mathrm{PKM} 2, \mathrm{M} 2$ isomer of pyruvate kinase; $\mathrm{LDH}$, lactate dehydrogenase; Oxa, oxaliplatin; siRNA, small interfering RNA; NC negative control.

(Fig. 4), demonstrating that glycolysis was suppressed. The results indicated that PKM2-siRNAs had greater effect on HCT116 cells.

PKM2-siRNA induced downregulation of glucose uptake and lactate production, which suggests a decline in cell metabolism. While the above results indicated that the difference in glycolysis metabolism between PKM2-siRNAs + oxaliplatin and oxaliplatin was not as profound as the difference between cell proliferation and apoptosis. It was hypothesized that there might be other glucose metabolic pathways closely associated with glycolysis which induce apoptosis.

It is known that the production of ROS can cause cellular damage and severe cytotoxicity, which can induce cell apoptosis (31). While NADPH, a metabolite of the PPP (pentose phosphate pathway), converts GSSH to GSH, which is the main free-radical scavenger neutralizing intracellular ROS (3) and then decrease ROS-induced apoptosis (32). Therefore, PKM2-siRNAs were postulated to increase ROS level through the suppression of PPP.

As expected, ROS levels were increased in the PKM2-siRNAs groups compared with the NC group (Fig. 5A and B). Subsequently, whether transfecting HCT116 cell lines with PKM2-siRNA attenuated GSH levels was investigated. As indicated in Fig. 4C, the GSH level in the PKM2-siRNAs groups was lower compared with that in the NC group.

Subsequently, the changes in the PPP (pentose phosphate pathway) were examined. As G6PD is a key enzyme involved in PPP, which converts glucose-6-phosphate to 6 Gluconolactone 6 phosphate (33), RT-qPCR was used to detect the change of mRNA level of G6PD following the transfection of PKM2-si155 into HCT116 cells. The results indicated that the average mRNA expression level of G6PD was decreased 
A

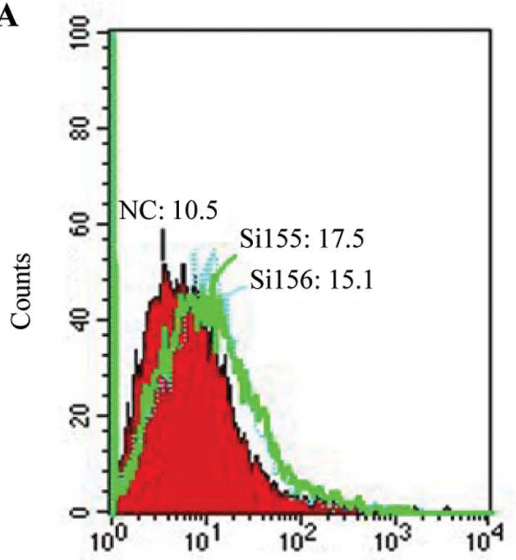

C

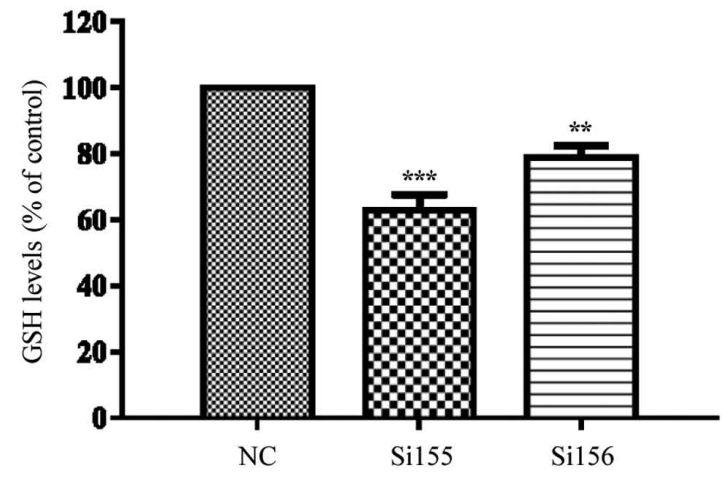

B

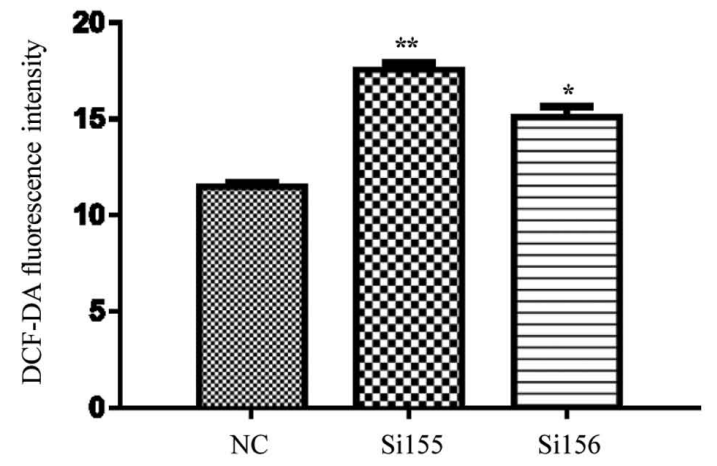

D

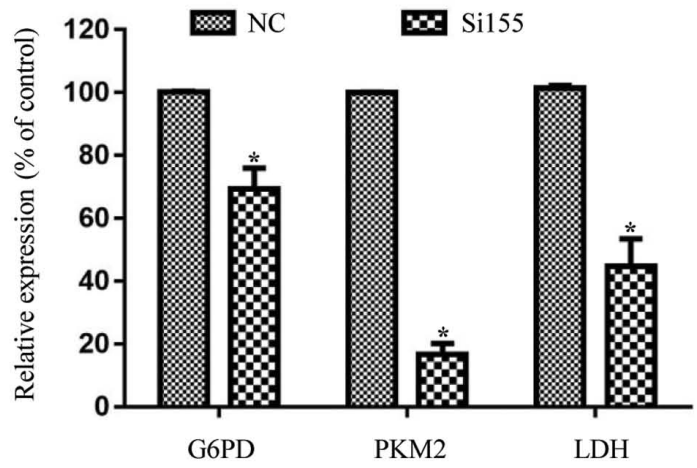

Figure 5. Changes in the pentose phosphate pathway redox parameters in PKM2-siRNAs transfected HCT116 cells. (A) Flow cytometric plots and (B) respective quantitative analysis of ROS levels. ROS levels following transfection with different PKM2-siRNAs were significantly increased compared with the NC group. (C) GSH levels in the PKM2-siRNAs groups were lower compared with the NC group. (D) mRNA expression levels of PKM2, G6PD and LDH were significantly decreased compared with the $\mathrm{NC}$ group. ${ }^{*} \mathrm{P}<0.05,{ }^{* * *} \mathrm{P}<0.01,{ }^{* * *} \mathrm{P}<0.001$ vs. respective NC. PKM2, M2 isomer of pyruvate kinase; siRNA, small interfering RNA; ROS, reactive oxygen species; GSH, glutathione; NC, negative control; LDH, lactate dehydrogenase; DCFDA, 2',7'-dichlorofluorescin diacetate.

compared with the NC group, which was consistent with PKM2-si155 and LDH (Fig. 5D).

\section{Discussion}

PKM2 is highly expressed in tumors compared with normal tissues (2). Aerobic glycolysis is the primary metabolic pathway used by cancer cells, and PKM2 is a key enzyme involved in this pathway (1). Additionally, PKM2 promotes tumor growth through non-metabolic pathways, such as by influencing the cell cycle protein D1, POU domain, class 5, transcription factor 1, Myc, mTOR, and Mucin-1 (34-38). PKM2 and hypoxia-inducible factor (HIF)-1 also interact in tumor cells. HIF-1 upregulates the expression of PKM2, and PKM2 assists HIF-1 in activating hundreds of genes in downstream pathways to overcome and adjust to hypoxic conditions $(39,40)$; HIF-1 target genes include those encoding: The glucose transporter GLUT1, which increases glucose uptake; lactate dehydrogenase A (LDHA), which converts pyruvate to lactate; and pyruvate dehydrogenase kinase 1 (PDK1), which inactivates pyruvate dehydrogenase, thereby transporting pyruvate away from the mitochondria and inhibiting $\mathrm{O}_{2}$ consumption (41). Autophagy suppresses the function of PKM2 through acetylation (42). Thus, given the crucial role of PKM2 in tumors, targeting PKM2 may be an effective treatment strategy.
Previous studies have examined whether PKM2 can be used as a target in cancer treatment. Christofk et al (43) firstly demonstrated that lung cancer cell proliferation was significantly inhibited by suppression of PKM2. Subsequent cell based and nude mice animal model studies of cholangiocarcinoma (44) revealed that the suppression of PKM2 decreased the proliferation of cancer cells, and also suppressed the invasion and angiogenesis of tumors. The present study revealed that PKM2 suppression results in proliferation inhibition and it also showed that tumor cell apoptosis increased after knockdown of PKM2, which is in line with previous studies. In the A549 lung cancer cell line (21), PKM2 targeting or docetaxel-based treatment killed cancer cells, and their combination showed synergistic effects. In CRC, the PKM2 mRNA expression levels were associated with oxaliplatin efficacy, tumors with the lowest PK-M2 levels exhibited the lowest response rates (22). In previous studies, Ginés et al (45) reported novel non-glycolytic roles of PKM2 in response to genotoxic damage and proposes BMF as a possible target gene of PKM2. The present study explored the mechanism of PKM2 in oxidative damage induced by the glycolytic pathway when combined with oxaliplatin. Compared with the NC and oxaliplatin only groups, cellular proliferation was inhibited and apoptosis was significantly increased in the PKM2-siRNAs and PKM2-siRNAs + oxaliplatin groups. PKM2-siRNAs combined with oxaliplatin showed 
a synergistic effect in CRC cells. Oxaliplatin may have reduced proliferation and differentiation of cells by crosslinking with DNA and suppressing DNA synthesis (26). PKM2-siRNAs affected the major metabolic pathway used by cancer cells resulting in apoptosis. As a result, PKM2 and oxaliplatin serve significant roles in cell proliferation and metabolism, respectively, avoiding overlapping effects and explaining the synergistic effects of the co-treatment.

To examine how siPKM2 initiated apoptosis, glucose uptake and lactate secretion fluxes of NC, PKM2-siRNAs, and PKM2-siRNAs + oxaliplatin groups in HCT116 and DLD1 cells were determined. PKM2 knockdown reduced glucose uptake and lactate secretion in the PKM2-siRNAs and PKM2-siRNAs + oxaliplatin groups, demonstrating that glycolysis was suppressed. However, the difference in glycolysis metabolism between PKM2-siRNAs + oxaliplatin and oxaliplatin was not as profound as the difference between cell proliferation and apoptosis. The mechanism may involve ROS. In an aerobic environment, anoxia (46) and hypoxia-oxidation result in the production of large amounts of ROS (47), which can induce cell apoptosis (31). PKM2 enables tumor cells to escape from ROS damage (3). Anoxia also upregulates HIF-1, thus increasing PKM2 expression (40). ROS oxidizes PKM2 Cys358, leading glucose into the PPP and the production of large quantities of NADPH to neutralize ROS (3). As a result, intracellular ROS does not increase. Therefore, it may be hypothesized that once PKM2 was suppressed, the balance between PKM2 and ROS is disrupted, resulting in increased ROS-induced apoptosis. A previous study also shows that G6PD knockdown lowers NADPH levels and increases cellular susceptibility to oxidative stress (32). The mRNA level of G6PD was significantly decreased after suppression with PKM2-siRNAs, indicating that the PPP was inhibited. PKM2-si155 induces downregulation of glucose uptake and lactate production, which suggests a decline in cell metabolism (43). As a result, the downregulation of G6PD mRNA may be the result of changes in cell metabolism. As NADPH is a metabolite of the PPP and GSH is the downstream product of NADPH, GSH levels were evaluated. Although NADPH was not examined in the present study, the levels of GSH were determined. GSH, to some extent, is a downstream mediator of NADPH, which interacts with ROS. The results showed that the GSH levels in the PKM2-siRNAs groups were significantly lower compared with the NC group. Furthermore, to explore if there was a negative association between GSH and ROS levels, ROS levels were detected. The ROS levels in the si155 group were increased compared with the NC group. Thus, suppressing PKM2 led to a decrease in GSH, which was closely associated with increased ROS and cell apoptosis induction.

\section{Acknowledgements}

Not applicable.

\section{Funding}

This study was supported by grants from the Natural Science Foundation of Guangdong, China (grant no. 2015A030313010), Science and Technology Program of Guangzhou, China (grant no. 1563000305) and the National Natural Science Foundation of China (grant nos. 81272641 and 81572409).

\section{Availability of data and materials}

The datasets used and/or analyzed during the current study are available from the corresponding author on reasonable request.

\section{Authors' contributions}

LPX and YMH conceived and designed the study. CXY and WHL designed and performed the experiments. MZM, QY and WZH analyzed the data. CXY and WHL wrote, revised, and edited the manuscript. All authors have read and approved the final manuscript.

\section{Ethics approval and consent to participate}

Not applicable.

\section{Patient consent for publication}

Not applicable.

\section{Competing interests}

The authors declare that they have no competing interests.

\section{References}

1. Vander Heiden MG, Cantley LC and Thompson CB: Understanding the Warburg effect: The metabolic requirements of cell proliferation. Science 324: 1029-1033, 2009.

2. Lu Z: Nonmetabolic functions of pyruvate kinase isoform M2 in controlling cell cycle progression and tumorigenesis. Chin J Cancer 31: 5-7, 2012.

3. Anastasiou D, Poulogiannis G, Asara JM, Boxer MB, Jiang JK, Shen M, Bellinger G, Sasaki AT, Locasale JW, Auld DS, et al: Inhibition of pyruvate kinase M2 by reactive oxygen species contributes to cellular antioxidant responses. Science 334: 1278-1283, 2011.

4. Christofk HR, Vander Heiden MG, Wu N, Asara JM and Cantley LC: Pyruvate kinase M2 is a phosphotyrosine-binding protein. Nature 452: 181-186, 2008.

5. Gomez-Escudero J, Clemente C, Garcia-Weber D, Acín-Pérez R, Millán J, Enríquez JA, Bentley K, Carmeliet P and Arroyo AG: PKM2 regulates endothelial cell junction dynamics and angiogenesis via ATP production. Sci Rep 9: 15022, 2019.

6. Lin Y, Zhai H, Ouyang Y, Lu Z, Chu C, He Q and Cao X: Knockdown of PKM2 enhances radiosensitivity of cervical cancer cells. Cancer Cell Int 19: 129, 2019.

7. Liu VM, Howell AJ, Hosios AM, Li Z, Israelsen WJ and Vander Heiden MG: Cancer-associated mutations in human pyruvate kinase M2 impair enzyme activity. FEBS Lett 594: 646-664, 2020.

8. Gupta V and Bamezai RN: Human pyruvate kinase M2: A multifunctional protein. Protein Sci 19: 2031-2044, 2010.

9. Kress S, Stein A, Maurer P, Weber B, Reichert J, Buchmann A, Huppert $\mathrm{P}$ and Schwarz M: Expression of hypoxia-inducible genes in tumor cells. J Cancer Res Clin Oncol 124: 315-320, 1998.

10. Kumar Y, Tapuria N, Kirmani N and Davidson BR: Tumour M2-pyruvate kinase: A gastrointestinal cancer marker. Eur J Gastroenterol Hepatol 19: 265-276, 2007.

11. Schneider J and Schulze G: Comparison of tumor M2-pyruvate kinase (tumor M2-PK), carcinoembryonic antigen (CEA), carbohydrate antigens CA 19-9 and CA 72-4 in the diagnosis of gastrointestinal cancer. Anticancer Res 23: 5089-5093, 2003.

12. Zhang B, Chen JY, Chen DD, Wang GB and Shen P: Tumor type M2 pyruvate kinase expression in gastric cancer, colorectal cancer and controls. World J Gastroenterol 10: 1643-1646, 2004.

13. Hathurusinghe HR, Goonetilleke KS and Siriwardena AK: Current status of tumor M2 pyruvate kinase (tumor M2-PK) as a biomarker of gastrointestinal malignancy. Ann Surg Oncol 14: 2714-2720, 2007 
14. Ogawa H, Nagano H, Konno M,Eguchi H, Koseki J,Kawamoto K, Nishida $\mathrm{N}$, Colvin $\mathrm{H}$, Tomokuni A, Tomimaru Y, et al: The combination of the expression of hexokinase 2 and pyruvate kinase M2 is a prognostic marker in patients with pancreatic cancer. Mol Clin Oncol 3: 563-571, 2015

15. Zhao Y, Shen L, Chen X, Qian Y, Zhou Q, Wang Y, Li K, Liu M, Zhang S and Huang X: High expression of PKM2 as a poor prognosis indicator is associated with radiation resistance in cervical cancer. Histol Histopathol 30: 1313-1320, 2015.

16. Schneider J, Morr H, Velcovsky HG, Weisse $G$ and Eigenbrodt E: Quantitative detection of tumor M2-pyruvate kinase in plasma of patients with lung cancer in comparison to other lung diseases. Cancer Detect Prev 24: 531-535, 2000.

17. Hegele A, Varga Z, Kosche B, Stief T, Heidenreich A and Hofmann R: Pyruvate kinase type tumor M2 in urological malignancies. Urol Int 70: 55-58, 2003.

18. Ugurel S, Bell N, Sucker A, Zimpfer A, Rittgen W and Schadendorf D: Tumor type M2 pyruvate kinase (TuM2-PK) as a novel plasma tumor marker in melanoma. Int J Cancer 117: 825-830, 2005

19. Lüftner D, Mesterharm J, Akrivakis C, Geppert R, Petrides PE, Wernecke KD and Possinger K: Tumor type M2 pyruvate kinase expression in advanced breast cancer. Anticancer Res 20 (6D): D5077-D5082, 2000.

20. Lin Y, Lv F, Liu F, Guo X, Fan Y, Gu F, Gu J and Fu L: High expression of pyruvate kinase M2 is associated with chemosensitivity to epirubicin and 5-fluorouracil in breast cancer J Cancer 6: 1130-1139, 2015.

21. Shi HS, Li D, Zhang J, Wang YS, Yang L, Zhang HL, Wang XH, Mu B, Wang W, Ma Y, et al: Silencing of pkm2 increases the efficacy of docetaxel in human lung cancer xenografts in mice. Cancer Sci 101: 1447-1453, 2010.

22. Martinez-Balibrea E, Plasencia C, Ginés A, Martinez-Cardús A, Musulén E, Aguilera R, Manzano JL, Neamati N and Abad A: A proteomic approach links decreased pyruvate kinase M2 expression to oxaliplatin resistance in patients with colorectal cancer and in human cell lines. Mol Cancer Ther 8: 771-778, 2009.

23. Shiragami R, Murata S, Kosugi C, Tezuka T, Yamazaki M, Hirano A, Yoshimura Y, Suzuki M, Shuto K and Koda K Enhanced antitumor activity of cerulenin combined with oxaliplatin in human colon cancer cells. Int J Oncol 43: 431-438, 2013

24. Gelibter AJ, Caponnetto S, Urbano F, Emiliani A, Scagnoli S, Sirgiovanni G, Napoli VM and Cortesi E: Adjuvant chemotherapy in resected colon cancer: When, how and how long? Surg Oncol 30: 100-107, 2019.

25. Petrelli F, Ghidini A and Zaniboni A: Cetuximab in association with an oxaliplatin-based chemotherapy as first-line treatment of metastatic colorectal cancer. Recenti Prog Med 108: 128-135, 2017 (In Italian)

26. Woynarowski JM, Faivre S, Herzig MC, Arnett B, Chapman WG Trevino AV, Raymond E, Chaney SG, Vaisman A, Varchenko M and Juniewicz PE: Oxaliplatin-induced damage of cellular DNA Mol Pharmacol 58: 920-927, 2000.

27. Goldberg MS and Sharp PA: Pyruvate kinase M2-specific siRNA induces apoptosis and tumor regression. J Exp Med 209: 217-224, 2012

28. Zhang H, Badur MG, Divakaruni AS, Parker SJ, Jäger C, Hiller K, Murphy AN and Metallo CM: Distinct metabolic states can support self-renewal and lipogenesis in human pluripotent stem cells under different culture conditions. Cell Rep 16 1536-1547, 2016.

29. Livak KJ and Schmittgen TD: Analysis of relative gene expression data using real-time quantitative PCR and the 2(T)(-Delta Delta C) method. Methods 25: 402-408, 2001.

30. Wils J: Adjuvant treatment of colon cancer: Past, present and future. J Chemother 19: 115-122, 2007.

31. Deng S, Yang Y, Han Y, Li X, Wang X, Li X, Zhang Z and Wang Y: UCP2 inhibits ROS-mediated apoptosis in A549 under hypoxic conditions. PLoS One 7: e30714, 2012.
32. Ju HQ, Lu YX, Wu QN, Liu J, Zeng ZL, Mo HY, Chen Y, Tian T, Wang Y, Kang TB, et al: Disrupting G6PD-mediated Redox homeostasis enhances chemosensitivity in colorectal cancer. Oncogene 36: 6282-6292, 2017.

33. Stanton RC: Glucose-6-phosphate dehydrogenase, NADPH, and cell survival. IUBMB Life 64: 362-369, 2012.

34. Yang W, Xia Y, Ji H, Zheng Y, Liang J, Huang W, Gao X, Aldape $\mathrm{K}$ and $\mathrm{Lu} Z$ : Nuclear PKM2 regulates $\beta$-catenin transactivation upon EGFR activation. Nature 480: 118-122, 2011.

35. Lee J, Kim HK, Han YM and Kim J: Pyruvate kinase isozyme type M2 (PKM2) interacts and cooperates with Oct- 4 in regulating transcription. Int J Biochem Cell Biol 40: 1043-1054, 2008.

36. Ohkouchi S, Block GJ, Katsha AM, Kanehira M, Ebina M, Kikuchi T, Saijo Y, Nukiwa T and Prockop DJ: Mesenchymal stromal cells protect cancer cells from ROS-induced apoptosis and enhance the Warburg effect by secreting STC1. Mol Ther 20: 417-423, 2012.

37. Sun $Q$, Chen $X$, Ma J, Peng $H$, Wang F, Zha X, Wang $Y$, Jing Y, Yang $\mathrm{H}$, Chen $\mathrm{R}$, et al: Mammalian target of rapamycin up-regulation of pyruvate kinase isoenzyme type M2 is critical for aerobic glycolysis and tumor growth. Proc Natl Acad Sci USA 108: 4129-4134, 2011.

38. Kosugi M, Ahmad R, Alam M, Uchida Y and Kufe D: MUC1-C oncoprotein regulates glycolysis and pyruvate kinase $\mathrm{M} 2$ activity in cancer cells. PLoS One 6: e28234, 2011.

39. Luo W and Semenza GL: Pyruvate kinase M2 regulates glucose metabolism by functioning as a coactivator for hypoxia-inducible factor 1 in cancer cells. Oncotarget 2: 551-556, 2011.

40. Luo W, Hu H, Chang R, Zhong J, Knabel M, O'Meally R, Cole RN, Pandey A and Semenza GL: Pyruvate kinase M2 is a PHD3-stimulated coactivator for hypoxia-inducible factor 1. Cell 145: 732-744, 2011.

41. Wheaton WW and Chandel NS: Hypoxia. 2. Hypoxia regulates cellular metabolism. Am J Physiol Cell Physiol 300: C385-C393, 2011.

42. Lv L, Li D, Zhao D, Lin R, Chu Y, Zhang H, Zha Z, Liu Y, Li Z, $\mathrm{Xu} \mathrm{Y,} \mathrm{et} \mathrm{al:} \mathrm{Acetylation} \mathrm{targets} \mathrm{the} \mathrm{M2} \mathrm{isoform} \mathrm{of} \mathrm{pyruvate}$ kinase for degradation through chaperone-mediated autophagy and promotes tumor growth. Mol Cell 42: 719-730, 2011.

43. Christofk HR, Vander Heiden MG, Harris MH, Ramanathan A Gerszten RE, Wei R, Fleming MD, Schreiber SL and Cantley LC: The M2 splice isoform of pyruvate kinase is important for cancer metabolism and tumour growth. Nature 452: 230-233, 2008.

44. Yu G, Yu W, Jin G, Xu D, Chen Y, Xia T, Yu A, Fang W, Zhang X, $\mathrm{Li} Z$ and $\mathrm{Xie} \mathrm{K}$ : PKM2 regulates neural invasion of and predicts poor prognosis for human hilar cholangiocarcinoma. Mol Cancer 14: 193, 2015.

45. Ginés A, Bystrup S, Ruiz de Porras V, Guardia C, Musulén E, Martínez-Cardús A, Manzano JL, Layos L, Abad A and Martínez-Balibrea E: PKM2 subcellular localization is involved in oxaliplatin resistance acquisition in HT29 human colorectal cancer cell lines. PLoS One 10: e0123830, 2015.

46. Solaini G, Baracca A, Lenaz G and Sgarbi G: Hypoxia and mitochondrial oxidative metabolism. Biochim Biophys Acta 1797: 1171-1177, 2010.

47. Collins P, Jones C, Choudhury S, Damelin L and Hodgson H: Increased expression of uncoupling protein 2 in HepG2 cells attenuates oxidative damage and apoptosis. Liver Int 25: 880-887, 2005.

This work is licensed under a Creative Commons Attribution-NonCommercial-NoDerivatives 4.0 International (CC BY-NC-ND 4.0) License. 